\title{
Dark Matter Annihilation Can Produce a Detectable Antihelium Flux through $\bar{\Lambda}_{b}$ Decays
}

\author{
Martin Wolfgang Winkler $\odot^{*}$ and Tim Linden $\odot^{\dagger}$ \\ Stockholm University and The Oskar Klein Centre for Cosmoparticle Physics, Alba Nova, 10691 Stockholm, Sweden
}

(Received 10 July 2020; accepted 11 February 2021; published 8 March 2021)

\begin{abstract}
Recent observations by the Alpha Magnetic Spectrometer (AMS-02) have tentatively detected a handful of cosmic-ray antihelium events. Such events have long been considered as smoking-gun evidence for new physics, because astrophysical antihelium production is expected to be negligible. However, the darkmatter-induced antihelium flux is also expected to fall below current sensitivities, particularly in light of existing antiproton constraints. Here, we demonstrate that a previously neglected standard model processthe production of antihelium through the displaced-vertex decay of $\bar{\Lambda}_{b}$-baryons - can significantly boost the dark matter induced antihelium flux. This process can entirely dominate the production of high-energy antihelium nuclei, increasing the rate of detectable AMS-02 events by 2 orders of magnitude.
\end{abstract}

DOI: 10.1103/PhysRevLett.126.101101

Introduction.-The detection of massive cosmic-ray antinuclei has long been considered a holy grail for WIMP dark matter searches [1,2]. Primary cosmic rays mostly consist of protons and nuclei that are accelerated by astrophysical sources like supernovae. Secondary cosmic rays produced from the hadronic interactions of primary cosmic rays can include an antinuclei component, but the flux is highly suppressed by baryon number conservation and kinematic constraints [3,4]. Dark matter annihilation, on the other hand, occurs within the rest frame of the Milky Way and produces equal baryon and antibaryon fluxes [1,5-7]

The preeminent target for cosmic-ray antinucleon searches is antideuterium, which inhabits a "goldilocks zone" where the specificity of the dark matter signal is high and the flux is potentially detectable [5]. Significant satellite- [8] and balloon-based [9,10] efforts have been undertaken to detect these antideuterons. More recently, ${ }^{3} \overline{\mathrm{He}}$ has also been considered as a dark matter detection target, though even optimistic models based on showeraveraged nucleon statistics indicate that the ${ }^{3} \overline{\mathrm{He}}$ flux should fall far below that of $\overline{\mathrm{d}}[11,12]$.

Recently, however, the Alpha Magnetic Spectrometer (AMS-02) has announced the potential detection of $\mathcal{O}(10)$ ${ }^{3} \overline{\mathrm{He}}$ events, along with $\sim 2$ potential ${ }^{4} \overline{\mathrm{He}}$ events [13]. Such a detection could revolutionize dark matter searches, as the probability of obtaining this signal from astrophysics is vanishingly small $[3,12-19]$. Unfortunately, dark matter models are also expected to produce $\ll 1$ detectable antihelium event $[11,12]$. Because the verified detection

Published by the American Physical Society under the terms of the Creative Commons Attribution 4.0 International license. Further distribution of this work must maintain attribution to the author(s) and the published article's title, journal citation, and DOI. Funded by SCOAP. of cosmic-ray antihelium would provide bulletproof evidence of new physics, several studies have investigated adaptations to boost the antihelium rate, including changes to the coalescence momentum of ${ }^{3} \overline{\mathrm{He}}$ [20], the effects of cosmic-ray reacceleration [21], extended dark sectors [22], or exotic sources like primordial antimatter clouds [18].

In this Letter, we challenge the current understanding that standard dark matter annihilation cannot produce a measurable antihelium flux. Our analysis examines a known, and potentially dominant, antinuclei production mode that has been neglected by previous literature-the production of antihelium through the displaced-vertex decays of the $\bar{\Lambda}_{b}$.

The physical intuition behind our model is as follows. $\bar{\Lambda}_{b}$ baryons (and other unstable bottom hadrons) are generically produced in dark matter annihilation channels involving $b$ quarks. However, $\bar{\Lambda}_{b}$ is uniquely important because it has (i) an antibaryon number, and (ii) a $5.6 \mathrm{GeV}$ rest mass which lies just above the $4.7 \mathrm{GeV}$ rest mass of three stable antibaryons and two stable baryons (e.g., two antiprotons, an antineutron, and two protons). Thus-if a $\bar{\Lambda}_{b}$ decays to such a state- the constituent particles have small relative momenta in the $\bar{\Lambda}_{b}$ rest frame, allowing the constituent antiparticles to efficiently coalesce into antihelium. Intriguingly, because any ${ }^{3} \overline{\mathrm{He}}$ produced by $\bar{\Lambda}_{b}$ inherits its boost factor, these antinuclei can obtain the large center-ofmass momenta necessary to fit AMS-02 data [13].

State of the field.-We first examine why such decays have been previously neglected. Dark matter is usually assumed to annihilate into quark or gluon states. Their subsequent hadronization produces a cascade of (meta)stable particles including antiprotons and antineutrons. Rarely, these antiparticles may fuse into heavier antinuclei. Because of the complexity of antinuclei formation, models typically employ a "coalescence condition," where antiparticles formed with relative momenta below a coalescence 
momentum $p_{c}$ are assumed to combine into heavier antinuclei [23]. The value of $p_{c}$ is tuned to collider data.

Because of the small antideuterons and antihelium production rates, most analyses follow an "event-averaged" approach. These studies first calculate the fluxes of individual antiprotons and antineutrons, and subsequently cross match these particles between events to calculate the antinuclei yield. This produces precise spectral measurements, but assumes that individual antiparticle momenta are uncorrelated-an assumption that is strongly violated by displaced-vertex decays.

Several studies have also investigated coalescence in an event-by-event framework [11,12,24]. However, these analyses have exclusively focused on prompt antinucleus production at the initial vertex. Antinuclei originating from long-lived intermediate resonances have been rejected to prevent cross mixing between particles produced with similar momenta but different phase-spaces. As a consequence, the decay of single intermediate particles into multinucleon final states has been ignored.

Methodology.-In this study, we examine the antihelium flux from dark matter annihilation on an event-by-event basis. We improve upon previous techniques by including contributions from multiple antiparticle states produced at single displaced vertices.

To derive the antihelium spectrum, we employ two state-of-the-art event generators: PYTHIA (version 8.2) and Herwig (version 7.2). Herwig offers the option to carry out $\Lambda_{b}$ decays with the specialized tool EvtGen, which we also consider. (By default, Herwig uses EvtGen for $B$ decays but its own cluster hadronization algorithm for $\Lambda_{b}$ decays.) While Herwig reproduces LEP measurements of the transition ratio $f\left(b \rightarrow \Lambda_{b}\right)=0.1_{-0.03}^{+0.04}[25,26]$ within $1 \sigma$, PYTHIA falls short by a factor $\sim 3$ (see Ref. [27] for the calibration of the PYTHIA model parameters which determine the $\Lambda_{b}$ spectrum). Thus, we also consider a tuned version of PYTHIA (denoted " $\Lambda_{b}$ tune") where we increase diquark formation in hadronization (probQQtoQ) to match LEP data.

Nucleus formation is implemented via an event-by-event coalescence model, which takes the coalescence momentum $p_{c}$ as a free parameter. While $p_{c}$ is well defined for two-particle states like antideuterium, several definitions of $p_{c}$ exist for multiparticle states like antihelium. Reference [12] requires that all three particle pairs have a momentum difference $\left|p_{i}-p_{j}\right|<p_{c}$, while Ref. [11] requires that all three particles lie within a sphere of radius $p_{c} / 2$. These models produce antihelium fluxes that differ by $\sim 15 \%$, which can be accounted for by normalizing $p_{c}$ to fit ALICE data.

Here, we assume antihelium nuclei merge if all particles lie within a momentum sphere of radius $2^{2 / 6} p_{c} / 2$. The extra factor of $2^{1 / 6}$, compared to Ref. [11], is required to match the definition of $p_{c}$ in the analytic coalescence model (see Supplemental Material [28]). In addition to the coalescence condition, we require antinucleons to either originate from the initial vertex or from the same parent particle vertex (most importantly the $\bar{\Lambda}_{b}$ ).

We determine $p_{c}$ separately for each PYTHIA and Herwig implementation via a fit to ALEPH antideuteron data [29] and ALICE antihelium data [30] (see Supplemental Material [28] and [31-33]):

$p_{c}=239_{-30}^{+25} \mathrm{MeV} \quad$ (Pythia),

$p_{c}=124_{-16}^{+13} \mathrm{MeV} \quad\left(\right.$ Pythia $\Lambda_{b}$ tune $)$,

$p_{c}=215_{-30}^{+25} \mathrm{MeV} \quad$ (Herwig, Herwig + EvtGen).

Notice that the diquark parameter in PYTHIA also significantly boosts prompt antinucleus production. This is compensated by reducing the coalescence momentum in the $\Lambda_{b}$-tune model by a factor $\sim 0.6$.

The key results of our analysis are independent of dark matter mass. However, the antihelium spectrum depends on the boost-factor imparted to the $\bar{\Lambda}_{b}$, which depends on the dark matter mass and final state. As a benchmark scenario, we take $m_{\chi}=67 \mathrm{GeV},\langle\sigma v\rangle=2 \times 10^{-26} \mathrm{~cm}^{3} / \mathrm{s}$ and assume that dark matter annihilates into bottom quarks. These choices are motivated by the Galactic center excess and antiproton excess, which use similar dark matter parameters [34-36]. Additionally, we examine a tuned scenario, where an $80 \mathrm{GeV}$ dark matter particle annihilates through light mediators $(\phi)$, with masses of $14 \mathrm{GeV}$, which subsequently decay to $b \bar{b}$ final states. This scenario places a significant fraction of the particle energy directly above the $\bar{\Lambda}_{b}$ mass, increasing the antihelium production rate. While we refrain from a detailed fit to AMS-02 antiproton data, we note that our coalescence modeling does not affect the antiproton flux, and our models predict similar antiproton yields as previous fits to AMS-02 data [35-37].

To calculate the local antihelium flux and spectrum, we must account for antihelium propagation through the Galactic halo. Because such propagation is not the focus of our study, we employ a standard two-zone diffusion model fit to AMS-02 B/C and antiproton data [37,38] (see Table 3 in Ref. [37]). We normalize the dark matter flux using an NFW profile [39] with a local density of $0.38 \mathrm{GeV} \mathrm{cm}^{-3}$ [40-42]. The local propagation of lowenergy cosmic rays is severely affected by the heliosphere, which we account for using an improved force-field description [43] that includes charge-sign effects. We note that tritons decay to antihelium before reaching Earth, and treat this contribution additively to determine the antihelium flux.

Finally, we note that astrophysical sources also produce antihelium through the $\bar{\Lambda}_{b}$ channel. However, inclusive bottom production rates at the LHC [44], indicate a $\mathcal{O}(0.1 \mathrm{nb})$ cross section for displaced antihelium emission through $\bar{\Lambda}_{b}$ in proton-proton collisions; compared to the prompt antihelium cross section of $\mathcal{O}(10 \mathrm{nb})$ [30]. 


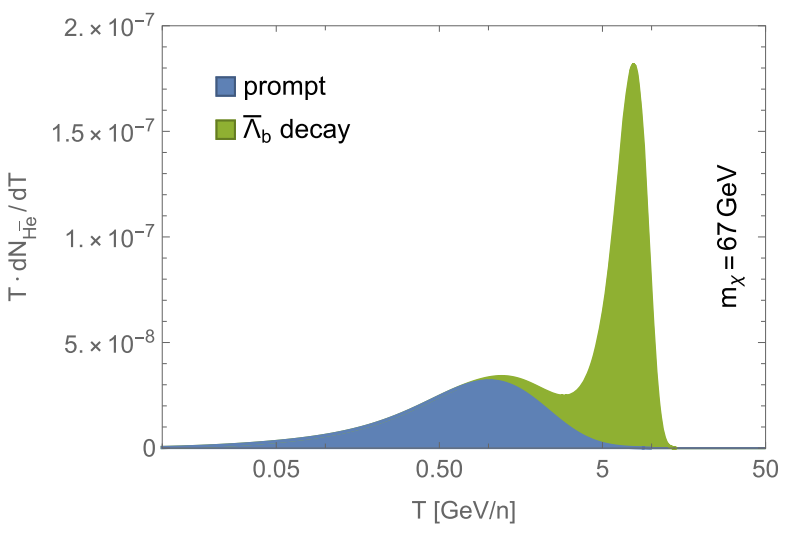

FIG. 1. Antihelium injection spectrum from dark matter annihilation using the PYTHIA event generator. The prompt and $\bar{\Lambda}_{b^{-}}$ induced contributions are shown separately. The antihelium flux from $\bar{\Lambda}_{b}$ decays exceeds that of prompt events by nearly a factor of 2 , and entirely dominates the production of high-energy antihelium.

At sub-LHC energies the suppression of the displaced component will be even stronger. Hence, in contrast to dark matter, the effect of the $\bar{\Lambda}_{b}$ channel on the astrophysical antihelium flux should be below $1 \%$. We do not consider this background further.

Results.-In Fig. 1, we plot the antihelium injection spectrum from $67 \mathrm{GeV}$ dark matter annihilation using PYTHIA. Intriguingly, we find that displaced-vertex $\bar{\Lambda}_{b}$ decays produce the majority $(\sim 60 \%)$ of the antihelium flux. More importantly, the boost factor obtained by $\bar{\Lambda}_{b}$ is efficiently transferred to antihelium nuclei, dominating the high-energy regime where AMS-02 events are observed.

In Table I and Fig. 2 we plot the integrated and differential antihelium flux at Earth. For most models, the addition of $\bar{\Lambda}_{b}$ decays significantly increases the total event rate and predicts the detection of $\mathcal{O}(1)$ antihelium event in AMS-02 data. Interestingly, while the total antihelium flux increases by a factor $\sim 3$, the number of detectable antihelium events increases by more than an order of magnitude due to the increased sensitivity of AMS-02 and the decreasing effect of solar modulation at higher energies. We note that these results do not include the 20\%-70\% systematic uncertainties in the coalescence momentum $\left(p_{c}\right)$, or systematic uncertainties related to cosmic-ray propagation. We stress that changes in the propagation parameters do not significantly alter the $\overline{\mathrm{He}} / \bar{p}$ ratio. Since the antiproton spectrum is strongly constrained by data [45], the corresponding propagation uncertainty in the antihelium flux will be small.

In the PYTHIA $\Lambda_{b}$-tune and Herwig + EvtGen models, the predicted antihelium flux increases beyond a factor of 100. However, our default Herwig analysis does not efficiently produce antihelium through the $\bar{\Lambda}_{b}$ channel. This is initially surprising because Herwig produces a $\bar{\Lambda}_{b}$ flux that is $4 \times$ higher than the default PYTHIA implementation.
TABLE I. Expected number of ${ }^{3} \overline{\mathrm{He}}$ and $\overline{\mathrm{d}}$ events from dark matter annihilation with $10 \mathrm{yr}$ of AMS-02 data, for our four event generators. For default PYTHIA $(P)$, we list prompt events in brackets. While both PYTHIA and the Herwig + EvtGen model produce a significant enhancement to the antihelium flux, default Herwig $(H)$ models predict a smaller contribution.

\begin{tabular}{lcccc}
\hline \hline Generator & \multicolumn{1}{c}{$P$} & $P\left[\Lambda_{b}\right.$ tune $]$ & \multicolumn{1}{c}{$H$} & $H+$ EvtGen \\
\hline${ }^{3} \overline{\mathrm{He}}$ events & $0.1(0.007)$ & 0.9 & 0.003 & 0.3 \\
$\overline{\mathrm{d}}$ events & $3.7(3.5)$ & 4.2 & 1.7 & 2.1 \\
\hline \hline
\end{tabular}

However, while $\operatorname{Br}\left(\bar{\Lambda}_{b} \rightarrow \overline{\mathrm{He}}\right) \simeq 3 \times 10^{-6}$ in PYTHIA, this branching ratio resides below $10^{-9}$ in Herwig.

The significant difference between PYTHIA and Herwig is rooted in the underlying hadronization models. PYTHIA utilizes the Lund String model [48], while Herwig uses a cluster model from Ref. [49]. In both PYTHIA and Herwig, the off-shell weak decay

$$
\bar{\Lambda}_{b} \rightarrow \bar{d} u \bar{u}(\overline{u d})_{0}
$$

is the dominant channel for displaced antihelium production, where $(\overline{u d})_{0}$ denotes an antidiquark. The branching fraction to this final state is $5 \times$ smaller in Herwig than in PYTHIA. However, this explains only a small fraction of the difference.

Since antihelium carries baryon number -3 , two diquark-antidiquark pairs must be acquired from the vacuum during hadronization. In the string model, such pairs may arise at each factorization step via string breaking, whereas only quark-antiquark pairs are created in Herwig's cluster fissioning model. In Herwig, new diquarks can emerge in the final decay of clusters into hadrons. However, the cluster mass distribution of $\bar{\Lambda}_{b}$ decays kinematically suppresses multibaryon final states. Thus, the small phase space of antihelium formation via $\bar{\Lambda}_{b}$ isolates a regime in which the differences between baryon production in the string and cluster hadronization models are most stark. We also note that Herwig produces a larger fraction of events that lie above the coalescence threshold. This is linked to a smaller probability of pion-emission in $\bar{\Lambda}_{b}$ decays compared to PYTHIA.

While the detailed analysis of these $\Lambda_{b}$ decay models lies beyond the scope of this work, our preliminary analysis indicates that reasonable changes to the Herwig cluster hadronization model do not qualitatively affect our conclusions. Notably, scans of the PwtDIquark parameter (which affects the diquark production probability in cluster decays) and the utilization of the Kupco hadronization model only marginally affect antihelium formation. Thus our analysis strongly motivates further investigations into $\Lambda_{b}$ decay physics.

Even for the PYTHIA and Herwig + EvtGen analyses, the predicted antihelium flux lies below the tentative detection 

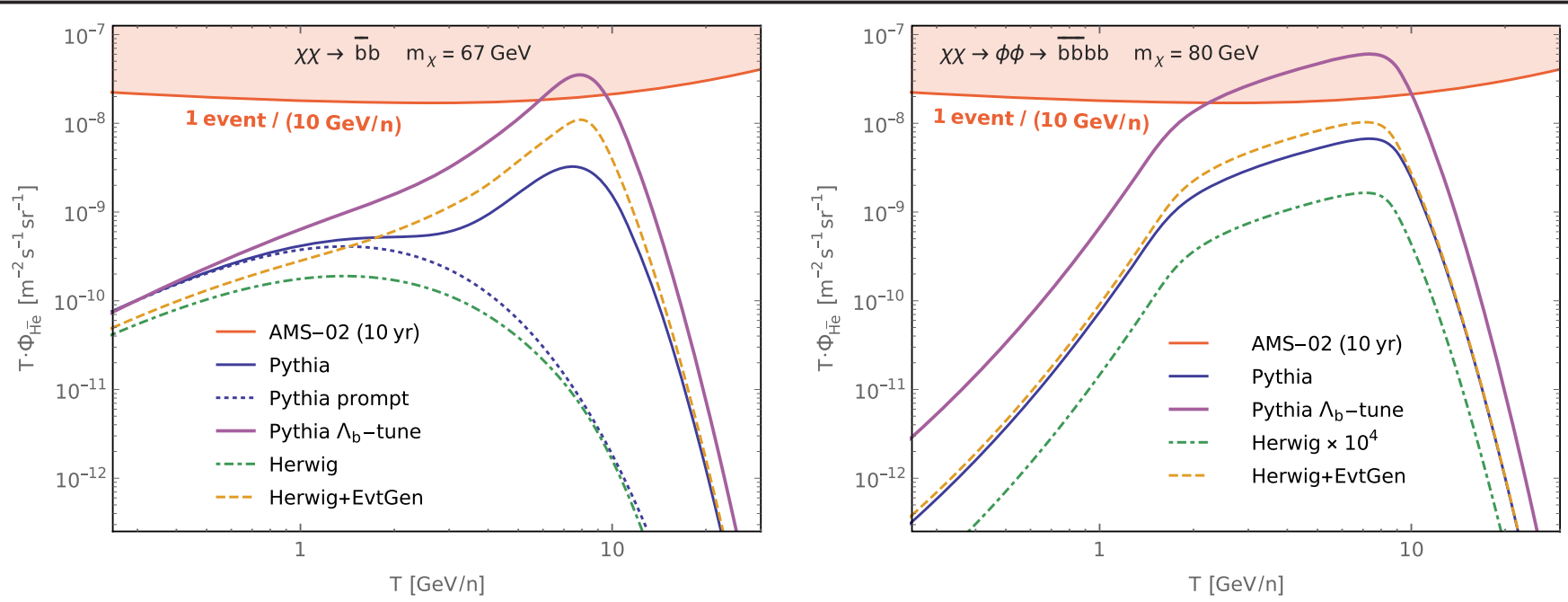

FIG. 2. (Left) Antihelium flux from dark matter annihilation with $\langle\sigma v\rangle=2 \times 10^{-26} \mathrm{~cm}^{3} / \mathrm{s}$, for event generators described in the text. The AMS-02 10-year sensitivity (orange) is derived from Refs. [46,47] (see Supplemental Material [28]). These results show that $\Lambda_{b}$ decays significantly boost the number of detectable antihelium events. (Right) Same, but for dark matter that annihilates through $14 \mathrm{GeV}$ light mediators. For this model, PYTHIA does not predict any prompt events (all events are produced by $\Lambda_{b}$ decay).

of $\mathcal{O}(10){ }^{3} \overline{\mathrm{He}}$ events [and potentially $\left.\mathcal{O}(1){ }^{4} \mathrm{He}\right]$ by AMS02. However, the significant boosts to antihelium production motivate the careful analysis of $\Lambda_{b}$ physics. Given the extreme variations in displaced antihelium production between event generators, it is conceivable that the true antihelium flux lies above the range obtained in this work. Furthermore, several additional mechanisms previously described in the literature (e.g., Alfvénic reacceleration [21]) could be coupled with our results to further enhance the antihelium flux. Finally, our analysis would predict a $\sim 10 \times$ larger antihelium event rate if we utilized the more optimistic antihelium sensitivity estimates of Ref. [21] (see Supplemental Material [28]).

Light-mediator models.-Nonstandard dark matter annihilation models can further enhance the $\bar{\Lambda}_{b}$ channel. One scenario focuses on dark matter annihilation into light mediators with masses just above the $\bar{\Lambda}_{b}$. This significantly increases the fraction of the dark matter energy that proceeds through the $\bar{\Lambda}_{b}$ channel. The mechanism is similar to previous leptophilic dark matter models, which include annihilations through a mediator [50,51]—except that our mediator decays into bottom pairs.

In Fig. 2 (right), we show the results for this model, adopting a dark matter mass of $80 \mathrm{GeV}$ and a mediator mass of $14 \mathrm{GeV}$. This scenario boosts the detectable antihelium flux by a factor of $\sim 3$, and further optimizes the $\bar{\Lambda}_{b}$ flux while diminishing the effect of prompt antihelium production.

Implications for antideuterons.-The goal of our analysis is to examine the effect of $\bar{\Lambda}_{b}$ baryon decay on

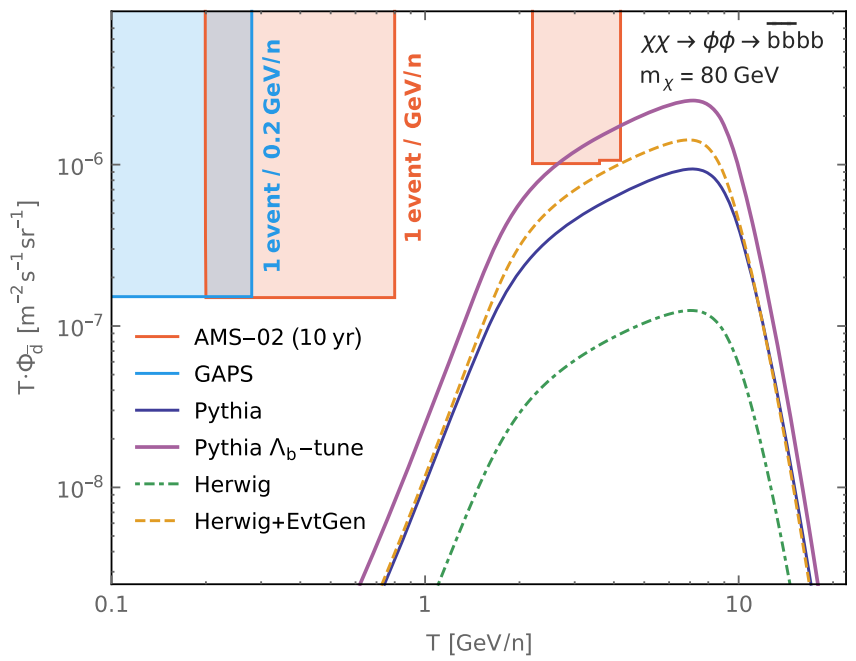

FIG. 3. Same as Fig. 2, but for antideuterons instead of antihelium. The AMS-02 and GAPS antideuteron sensitivities are taken from Ref. [10]. 
antihelium production. This is motivated by the tentative detection antihelium events by AMS-02. However, $\bar{\Lambda}_{b}$ decays can also produce antideuterons. As in the case of antihelium, the boost factor obtained by the $\bar{\Lambda}_{b}$ is imparted to the antideuterons, significantly enhancing the highenergy antideuteron flux.

In Table I and Fig. 3, we provide the antideuteron flux produced in our analysis, using the calculated antideuteron coalescence momenta (Supplemental Material [28]). We separate our results into prompt events and $\bar{\Lambda}_{b}$ baryons. Unlike antiheliums, antideuterons also obtain significant contributions from the displaced vertices of $B$ mesons ( $B$ mesons cannot decay into antihelium nuclei due to their $m_{B}=5.3 \mathrm{GeV}$ rest mass and vanishing baryon number). Since Herwig handles $B$ mesons through EvtGen by default, a small bump related to displaced decays is visible in the Herwig antideuteron spectrum. The reason that the high energy bump only marginally affects the antideuteron event rate in Table I is that it falls outside the AMS- 02 sensitivity window for the $m_{\chi}=67 \mathrm{GeV}$ dark matter mass that we considered (see Fig. 3).

Discussion.-In this Letter, we have shown that dark matter annihilations can produce a detectable antihelium flux. Our model assumes no new physics in the dark sector, but instead properly accounts for the contribution of displaced-vertex decays of $\bar{\Lambda}_{b}$ baryons produced in quark hadronization models. Thus, the results of this study should significantly impact the assumption that dark matter annihilation events typically produce a negligible antinuclei flux.

Moreover, our study significantly alters our understanding of antinuclei spectra from dark matter annihilation. Previous analytic and computational studies have focused on prompt antinuclei production $[1,11,12]$, predicting spectra that generically peak at $\lesssim 1 \mathrm{GeV}$. This spectrum has motivated the "silver-bullet" status of antinuclei searches as a background-free detection strategy, and driven experimental techniques to enhance the low-energy acceptance of balloon-based detectors, such as GAPS [10]. While our results do not spoil the potential of this strategy, our analysis provides the new opportunity to augment current searches with a brighter, high-energy spectral signature.

While our analysis predicts an enhancement of highenergy antihelium, the spectrum depends on the dark matter model. For example, $300 \mathrm{GeV}$ dark matter particles annihilating to $b \bar{b}$ produce a $\bar{\Lambda}_{b}$-induced antihelium flux which peaks at $\sim 30 \mathrm{GeV} / \mathrm{n}$. Conversely, for lighter dark matter, the antinuclei energy produced by $\bar{\Lambda}_{b}$ decays is reduced, potentially making the $\bar{\Lambda}_{b}$ induced antideuteron bump detectable by AMS-02. The relative normalization of the $\bar{\Lambda}_{b}$ contribution will also change. Since the transition ratio $f\left(\bar{b} \rightarrow \bar{\Lambda}_{b}\right)$ is energy independent, the number of antihelium nuclei produced through the $\bar{\Lambda}_{b}$ per dark matter annihilation is insensitive to the dark matter mass. The relative importance of the $\bar{\Lambda}_{b}$ channel slowly decreases toward higher dark matter masses due to the logarithmic increase of the prompt contribution.

Finally, while our results are generically produced by leading algorithms such as PYTHIA and EvtGen, it is notable that standard Herwig analyses produce a negligible (though nonzero) antihelium flux from $\bar{\Lambda}_{b}$ decay. We have identified the string and cluster hadronization models as the critical difference between these codes, and have found that the small kinematic window for $\bar{\Lambda}_{b}$ decays to antihelium nuclei isolates a regime where these approaches differ most acutely.

Furthermore, while colliders have illuminated several exclusive $\bar{\Lambda}_{b}$ decay pathways (see, e.g., Refs. [52,53]), no collider experiment has constrained the production of rare final states that may trigger antihelium formation. Our results motivate a dedicated program to investigate the decay properties of $\bar{\Lambda}_{b}$ baryons and understand the potential of displaced-vertex antinuclei searches.

We thank Ilias Cholis for helpful comments involving solar modulation potentials, Philip von Doetinchem for comments regarding the AMS-02 antihelium acceptance, and John Beacom, Mirko Boezio, Marco Cirelli, Fiorenza Donato, Carmelo Evoli, Dan Hooper, Alejandro Ibarra, Michael Korsmeier, Andrei Kounine, Alberto Oliva, Stefano Profumo, Pasquale Serpico, and Andrea Vittino for providing helpful comments on the manuscript. We especially thank Peter Reimitz and Peter Richardson for assistance with the Herwig code. T. L. is partially supported by the Swedish Research Council under Contract No. 2019-05135 and the Swedish National Space Agency under Contract No. 117/19. M. W. W. acknowledges support by the Vetenskapsrådet (Swedish Research Council) through Contract No. 638-2013-8993 and the Oskar Klein Centre for Cosmoparticle Physics.

*martin.winkler@su.se

†linden@fysik.su.se

[1] F. Donato, N. Fornengo, and P. Salati, Phys. Rev. D 62, 043003 (2000).

[2] P. von Doetinchem et al., J. Cosmol. Astropart. Phys. 08 (2020) 035.

[3] R. Duperray, B. Baret, D. Maurin, G. Boudoul, A. Barrau, L. Derome, K. Protasov, and M. Buenerd, Phys. Rev. D 71, 083013 (2005).

[4] D.-M. Gomez-Coral, A. Menchaca Rocha, V. Grabski, A. Datta, P. von Doetinchem, and A. Shukla, Phys. Rev. D 98, 023012 (2018).

[5] H. Baer and S. Profumo, J. Cosmol. Astropart. Phys. 12 (2005) 008.

[6] C. B. Brauninger and M. Cirelli, Phys. Lett. B 678, 20 (2009).

[7] Y. Cui, J. D. Mason, and L. Randall, J. High Energy Phys. 11 (2010) 017. 
[8] R. Battiston (AMS 02 Collaboration), Nucl. Instrum. Methods Phys. Res., Sect. A 588, 227 (2008).

[9] H. Fuke et al., Phys. Rev. Lett. 95, 081101 (2005).

[10] T. Aramaki, C. Hailey, S. Boggs, P. von Doetinchem, H. Fuke, S. Mognet, R. Ong, K. Perez, and J. Zweerink (GAPS Collaboration), Astropart. Phys. 74, 6 (2016).

[11] E. Carlson, A. Coogan, T. Linden, S. Profumo, A. Ibarra, and S. Wild, Phys. Rev. D 89, 076005 (2014).

[12] M. Cirelli, N. Fornengo, M. Taoso, and A. Vittino, J. High Energy Phys. 08 (2014) 009.

[13] A. Collaboration, AMS Days at La Palma, La Palma, Canary Islands, Spain, 2018.

[14] P. Chardonnet, J. Orloff, and P. Salati, Phys. Lett. B 409, 313 (1997).

[15] J. Herms, A. Ibarra, A. Vittino, and S. Wild, J. Cosmol. Astropart. Phys. 02 (2017) 018.

[16] K. Blum, Kenny Chun Yu Ng, R. Sato, and M. Takimoto, Phys. Rev. D 96, 103021 (2017).

[17] M. Korsmeier, F. Donato, and N. Fornengo, Phys. Rev. D 97, 103011 (2018).

[18] V. Poulin, P. Salati, I. Cholis, M. Kamionkowski, and J. Silk, Phys. Rev. D 99, 023016 (2019).

[19] A. Shukla, A. Datta, P. von Doetinchem, D.-M. GomezCoral, and C. Kanitz, Phys. Rev. D 102, 063004 (2020).

[20] A. Coogan and S. Profumo, Phys. Rev. D 96, 083020 (2017).

[21] I. Cholis, T. Linden, and D. Hooper, Phys. Rev. D 102, 103019 (2020).

[22] J. Heeck and A. Rajaraman, J. Phys. G 47, 105202 (2020).

[23] A. Schwarzschild and C. Zupancic, Phys. Rev. 129, 854 (1963).

[24] M. Kachelriess, S. Ostapchenko, and J. Tjemsland, J. Cosmol. Astropart. Phys. 08 (2020) 048.

[25] C. Caso et al. (Particle Data Group), Eur. Phys. J. C 3, 1 (1998).

[26] G. Abbiendi et al. (OPAL Collaboration), Eur. Phys. J. C 9 , 1 (1999).

[27] P. Skands, S. Carrazza, and J. Rojo, Eur. Phys. J. C 74, 3024 (2014).

[28] See Supplemental Material at http://link.aps.org/supplemental/ 10.1103/PhysRevLett.126.101101 for the derivation of coalescence momenta and for the antihelium sensitivity of AMS02 .

[29] S. Schael et al. (ALEPH Collaboration), Phys. Lett. B 639, 192 (2006).

[30] S. Acharya et al. (ALICE Collaboration), Phys. Rev. C 97, 024615 (2018).
[31] M. Kadastik, M. Raidal, and A. Strumia, Phys. Lett. B 683, 248 (2010).

[32] L. A. Dal and M. Kachelriess, Phys. Rev. D 86, 103536 (2012).

[33] N. Fornengo, L. Maccione, and A. Vittino, J. Cosmol. Astropart. Phys. 09 (2013) 031.

[34] T. Daylan, D. P. Finkbeiner, D. Hooper, T. Linden, S. K. N. Portillo, N. L. Rodd, and T. R. Slatyer, Phys. Dark Universe 12, 1 (2016).

[35] M.-Y. Cui, Q. Yuan, Y.-L. S. Tsai, and Y.-Z. Fan, Phys. Rev. Lett. 118, 191101 (2017).

[36] A. Cuoco, M. Kramer, and M. Korsmeier, Phys. Rev. Lett. 118, 191102 (2017).

[37] J. Heisig, M. Korsmeier, and M. W. Winkler, Phys. Rev. Research 2, 043017 (2020).

[38] A. Reinert and M. W. Winkler, J. Cosmol. Astropart. Phys. 01 (2018) 055.

[39] J. F. Navarro, C. S. Frenk, and S. D. White, Astrophys. J. 490, 493 (1997).

[40] R. Catena and P. Ullio, J. Cosmol. Astropart. Phys. 08 (2010) 004.

[41] M. Pato, F. Iocco, and G. Bertone, J. Cosmol. Astropart. Phys. 12 (2015) 001.

[42] P. de Salas, K. Malhan, K. Freese, K. Hattori, and M. Valluri, J. Cosmol. Astropart. Phys. 10 (2019) 037.

[43] I. Cholis, D. Hooper, and T. Linden, Phys. Rev. D 93, 043016 (2016).

[44] S. Chatrchyan et al. (CMS Collaboration), J. High Energy Phys. 04 (2012) 084.

[45] M. Aguilar et al. (AMS Collaboration), Phys. Rev. Lett. 117, 091103 (2016).

[46] A. Kounine, at Proceedings at ICRC 2011 (Local Organizing Committee of ICRC 2011, 2011).

[47] M. Aguilar et al. (AMS Collaboration), Phys. Rev. Lett. 115, 211101 (2015).

[48] B. Andersson, G. Gustafson, G. Ingelman, and T. Sjostrand, Phys. Rep. 97, 31 (1983).

[49] B. Webber, Nucl. Phys. B238, 492 (1984).

[50] N. Arkani-Hamed, D. P. Finkbeiner, T. R. Slatyer, and N. Weiner, Phys. Rev. D 79, 015014 (2009).

[51] M. Pospelov and A. Ritz, Phys. Lett. B 671, 391 (2009).

[52] A. M. Sirunyan et al. (CMS Collaboration), Phys. Lett. B 802, 135203 (2020).

[53] R. Aaij et al. (LHCb Collaboration), J. High Energy Phys. 09 (2019) 028. 